\title{
Is airway diameter measured accurately on routine axial CT scans? Comparison with true axial diameter using MPR in children with airway compression owing to pulmonary TB
}

\author{
Marie Grobbelaar, MB ChB, MMed (Rad Diag) \\ (deceased) \\ Department of Radiodiagnosis, Faculty of Health Sciences, University of \\ Stellenbosch Medical School \\ Savvas Andronikou, MB BCh, FCRad, FRCR \\ (Lond), PhD \\ Department of Radiology, University of of the Witwatersrand, Johannesburg
}

\section{Introduction}

Airway compression is a common complication of TB lymphadenopathy in children, ${ }^{1}$ and the diagnostic workup of patients with suspected tracheal or bronchial stenoses includes bronchoscopy and CT (computed tomography). ${ }^{2}$ This process affords the opportunity to study aspects of CT relating to airway stenosis. Axial CT scans produce excellent resolution in the horizontal plane, but the extent of airway disease may be underestimated if only axial images are obtained.

An advantage of using multidetector CT (MDCT) is the use of multiplanar reconstruction (MPR) to align the image along the longitudinal axis of the airway. There is also uncertainty if window settings affect the measurement of the airway diameter.

\section{Aim}

We wished to determine if there was a significant difference between the measurements of compressed airway diameter in the axial plane compared with measurements of diameter using MPR for determining longitudinal axis of the airway; and to evaluate how measurements on lung window settings compare with soft tissue window settings.

\section{Patients and methods}

For the study, 34 CT scans of children with central airway compression due to tuberculous lymphadenopathy were sourced from a digitally stored CT database (on CD) kept by a paediatric pulmonologist. The CT scans were all performed in one academic hospital according to a standard protocol, and were evaluated on a radiology workstation where MPR was performed. The maximum stenosis in the trachea, right main bronchus, bronchus intermedius and left main bronchus was measured, if present. The minimum diameter was taken on the axial scan as well as after MPR, providing the true longitudinal axis (Figs $1 \mathrm{~A}$ and $1 \mathrm{~B}$ ). All measurements were performed both in soft tissue window (Window 350/Level 50) and lung window (Window 1600/Level 500) settings (Figs
1C and 1D). Intraclass Correlation (ICC) was used for statistical analysis of the data.

\section{Results}

Scans of 18 male and 16 female children were evaluated. Their ages ranged from 1 month to 13.25 years (mean age 2.75 years). There were a total of 62 stenoses, comprising 14 in the trachea, 6 in the right main bronchus, 22 in the bronchus intermedius, and 20 in the left main bronchus. The average diameter of the narrowed airway size in the

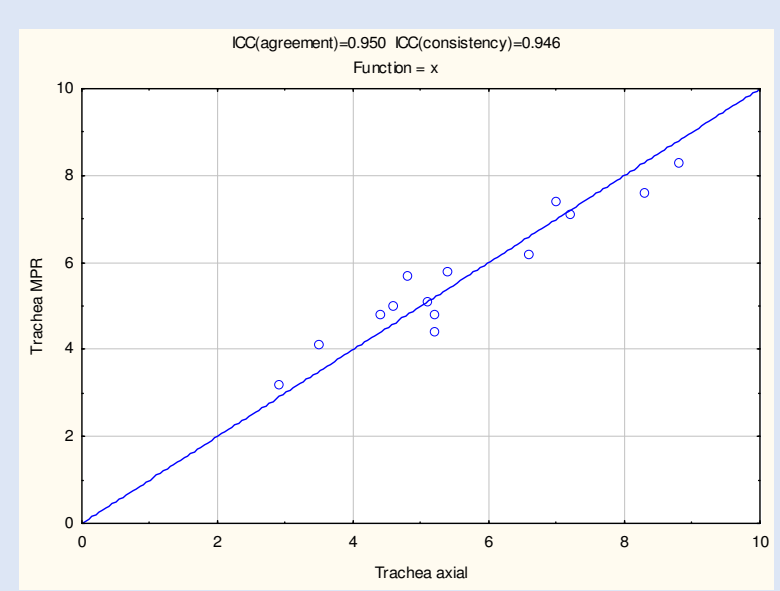

Graph 1. Comparison of axial measurements v. measurements after MPR in the trachea.

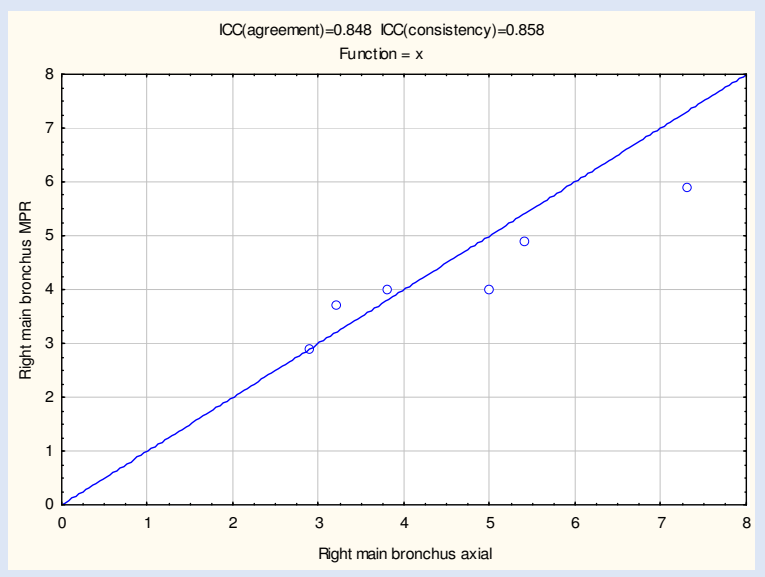

Graph 2. Comparison of axial measurements v. measurements after MPR in the right main bronchus. 


\section{ORIGINAL ARTICLE}

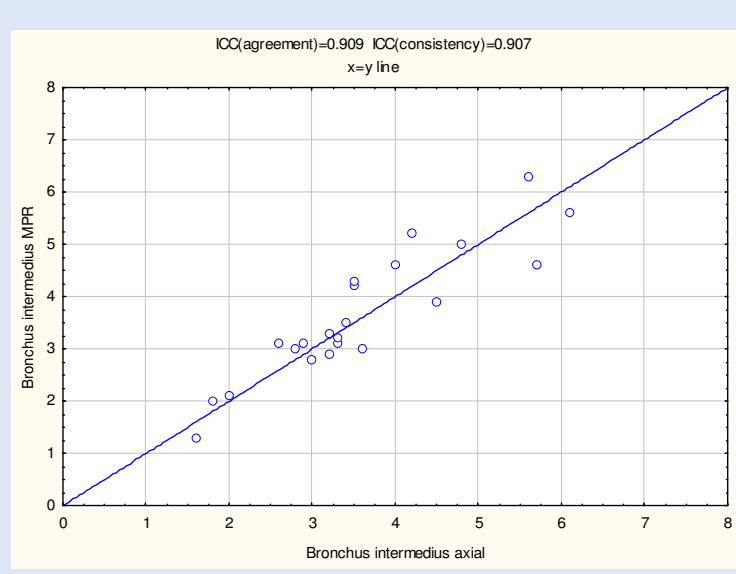

Graph 3. Comparison of axial measurements v. measurements after MPR in the bronchus intermedius.

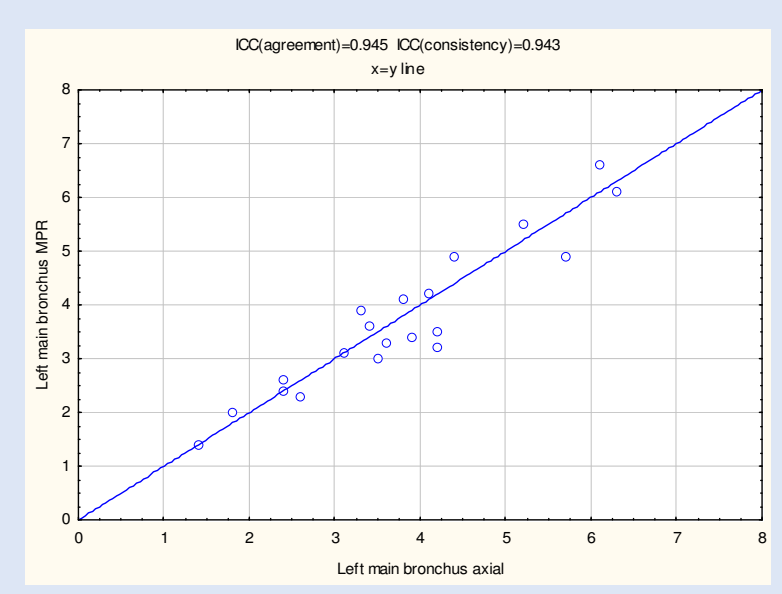

Graph 4. Comparison of axial measurements v. measurements after MPR in the left main bronchus.

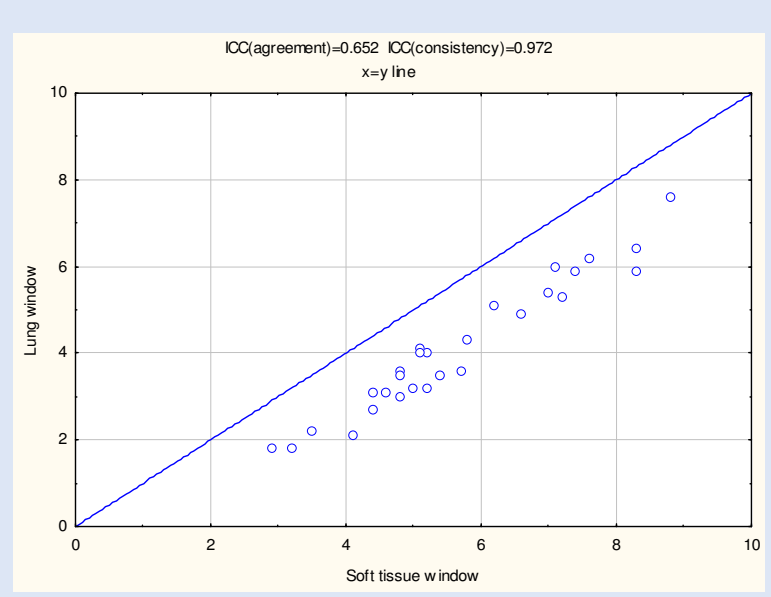

Graph 5. Comparison between measurements in soft tissue and lung windows in the trachea.

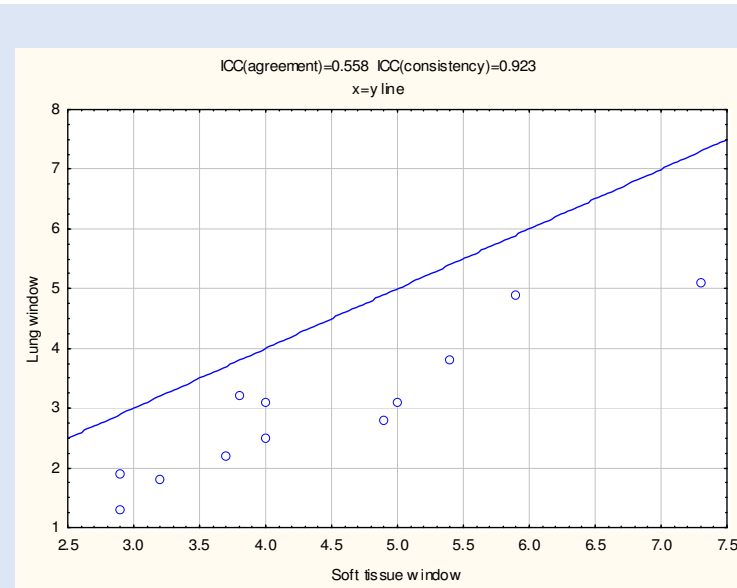

Graph 6. Comparison between measurements in soft tissue and lung windows in the right main bronchus.

selected children of this study was $4.2 \mathrm{~mm}$ (measured in soft tissue windows).

Graph 1 illustrates little difference (ICC agreement 0.950) between the sets of measurements of stenosis in the trachea, with a high consistency value (ICC consistency 0.946). Of the differences in measurements, $64.3 \%$ were $<0.5 \mathrm{~mm}, 35.7 \%$ were $0.5-1.0 \mathrm{~mm}$, and none was $>1 \mathrm{~mm}$.

Graph 2 illustrates the values of the 6 stenoses measured in the right main bronchus. The ICC values are lower (ICC agreement 0.848 and ICC consistency 0.858) compared with the figures for the trachea, bronchus intermedius and left main bronchus. Of the differences in measurements, $33.3 \%$ were $<0.5 \mathrm{~mm}, 33.3 \%$ were $0.5-1.0 \mathrm{~mm}$, and $33.3 \%$ were $>1 \mathrm{~mm}$.

Graph 3 illustrates little difference (ICC agreement 0.909) between the sets of measurements in the bronchus intermedius, with a high consistency value (ICC consistency 0.907). Of the differences in measurements, $54.5 \%$ were $<0.5 \mathrm{~mm}, 36.4 \%$ were $0.5-1.0 \mathrm{~mm}$, and $91 \%$ were $>1 \mathrm{~mm}$.

Graph 4 illustrates little difference (ICC agreement 0.945 ) between the sets of measurements in the left main bronchus, with a high consistency value (ICC consistency 0.943 ). Of the differences in measurements, $60 \%$ were $<0.5 \mathrm{~mm}, 35 \%$ weres $0.5-1.0 \mathrm{~mm}$, and $5 \%$ were $>1 \mathrm{~mm}$.

Table I. The difference in millimetres between axial measurements and measurements after MPR in the longitudinal axis (total number of stenoses $=62$ )

Difference

Trachea

Right main bronchus

Bronchus intermedius Left main bronchus Total

$<0.5$ mm
9
2
12
12
35

0.5 - $1.0 \mathrm{~mm}$

5

2

8

7

22

\section{$>1 \mathrm{~mm}$}

0

2

2

1

5 


\section{ORIGINAL ARTICLE}
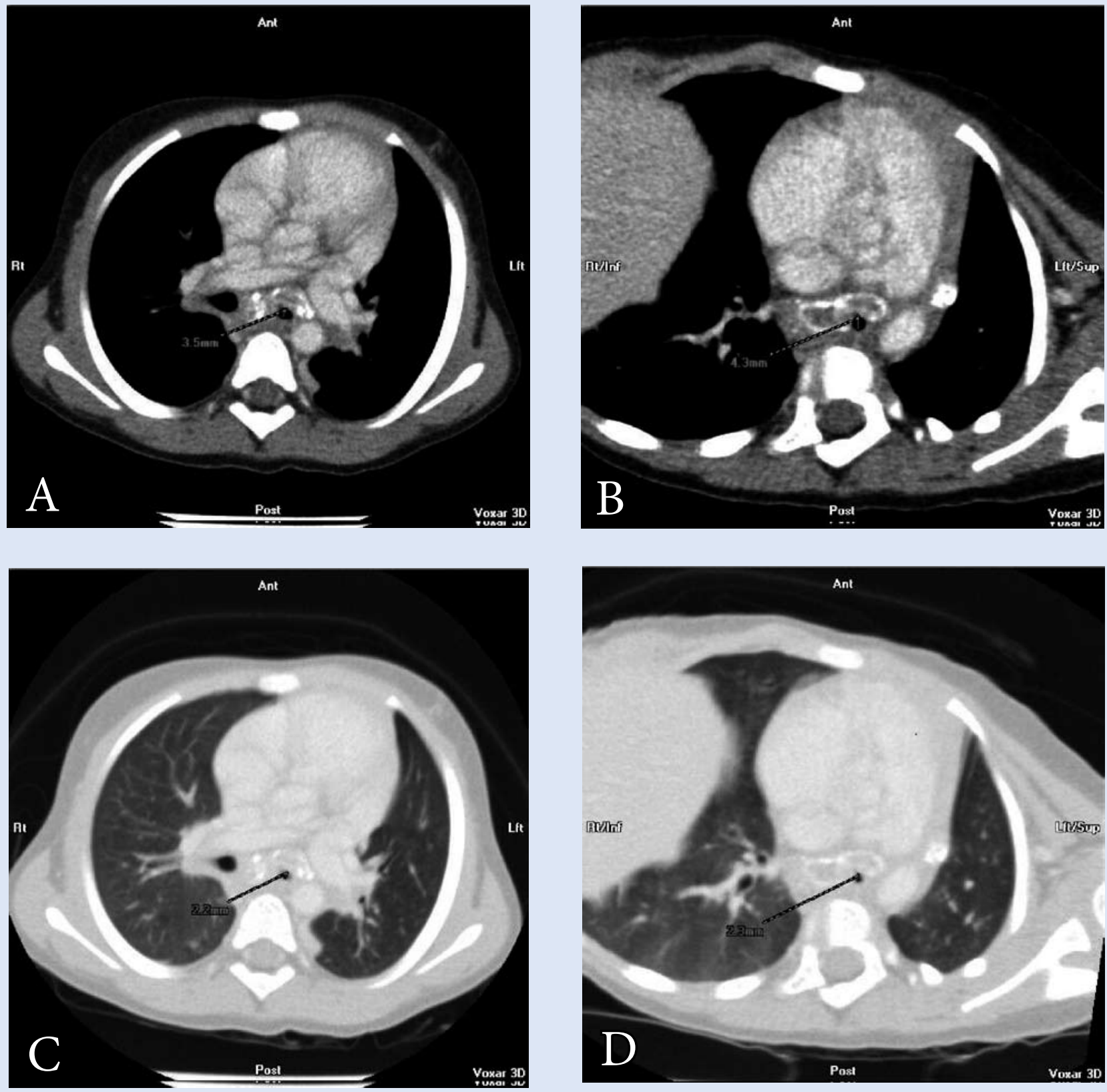

Fig. $1 A$ - D. MDCT of the chest in a 25-month-old boy with compression of the left main bronchus owing to tuberculous lymphadenopathy. A: Standard axial image in soft tissue windows illustrating the measurement at the maximum stenosis in the left main bronchus ( 3.5 mm). B: Measurement of diameter after MPR along the longitudinal axis of the airway $(4.3 \mathrm{~mm})$. Note the calcified lymphadenopathy around the airway. C: Image $A$ in lung windows, measurement $2.2 \mathrm{~mm}$. D: Image $B$ in lung windows, measurement $2.3 \mathrm{~mm}$.

Table I indicates the differences between axial measurements and those after MPR in the longitudinal axis for all the airways: of the differences, $56.4 \%$ were $<0.5 \mathrm{~mm}, 35.5 \%$ were $0.5-1.0 \mathrm{~mm}$, and $8.1 \%$ were $>1 \mathrm{~mm}$.

Graphs 5 and 6 illustrate a high consistency (ICC consistency of 0.972 for tracheal measurements and 0.923 for right main bronchus measurements) between values on soft tissue window and lung window for the trachea and right main bronchus. Lung window measurements in the trachea were $1.58 \mathrm{~mm}$ (root mean square error) less than measurements in soft tissue windows and $1.52 \mathrm{~mm}$ (root mean square error) less in the right main bronchus.

Graphs 7 and 8 also illustrate diameter measurements taken in the bronchus intermedius and left main bronchus on lung window and were consistently less than measurements in soft tissue windows (ICC consistency 0.889 for bronchus intermedius and 0.923 for left main bronchus). Here there was less difference for smaller diameter airways 


\section{ORIGINAL ARTICLE}

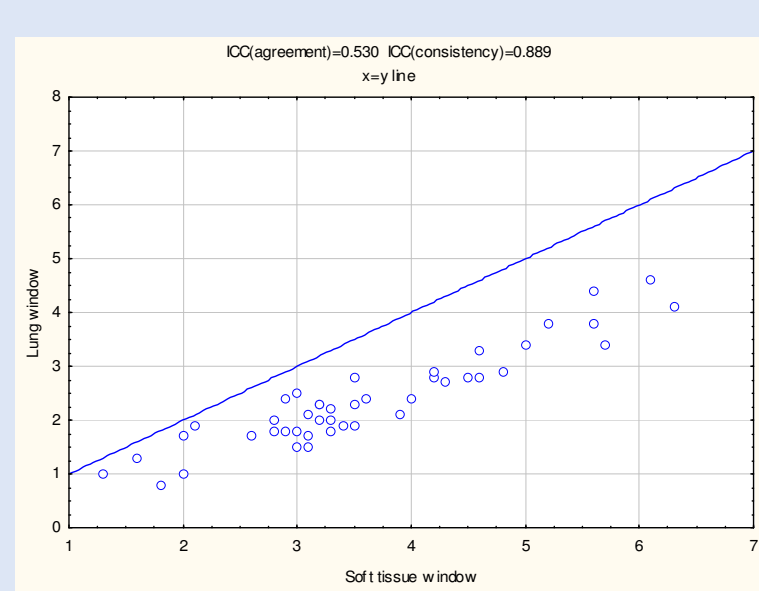

Graph 7. Comparison between measurements in soft tissue and lung windows in the bronchus intermedius.

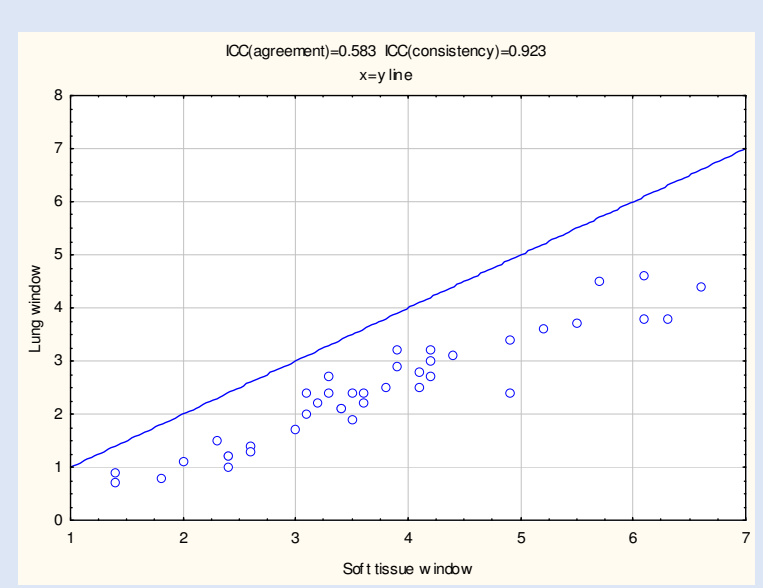

Graph 8. Comparison between measurements in soft tissue and lung windows in the left main bronchus.

(more severe stenoses) compared with differences for larger diameters. On average, the diameters were smaller by $1.4 \mathrm{~mm}$ on lung windows compared with soft tissue window at the same location.

\section{Discussion}

CT detects mediastinal, airway and lung abnormalities with high accuracy, sensitivity and specificity. ${ }^{3-5}$ The introduction of MDCT scanners has made it possible to acquire high-resolution images of the upper, central and segmental airways within a short acquisition time. ${ }^{6}$ In patients with airway compression, the advantage of CT over bronchoscopy is that the cause of airway compression can be diagnosed and the airway distal to the obstruction can be evaluated in areas that are usually inaccessible to bronchoscopy. Additional areas of stricture beyond an area of narrowing can also be evaluated. ${ }^{5,7}$ Chest CT is therefore an indispensable tool for identifying airway stenoses caused by TB lymphadenopathy.

Although axial CT images are sufficient for evaluating most airway abnormalities, there are inherent limitations of axial images for assessing the airways:

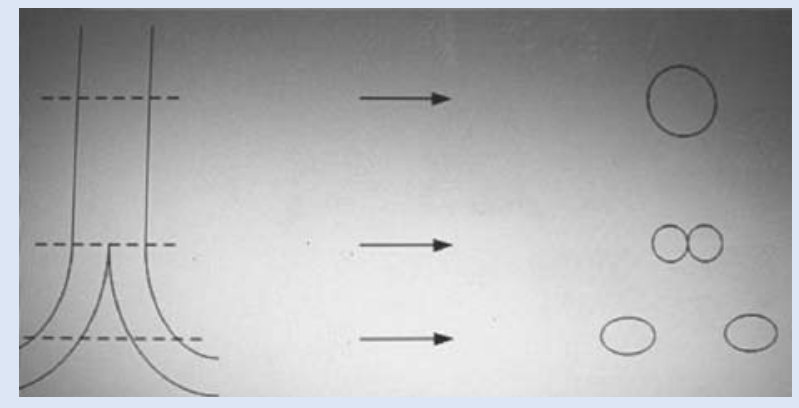

Fig 2. Illustration of axially imaged central airways. The cuts demonstrated are through the trachea, carina and main bronchi. An axial cut through the vertical trachea provides an accurate diameter of this tubular structure. If a more oblique structure is cut axially, the shape is oblong and may give an inaccurate measurement if there is asymmetrical compression.

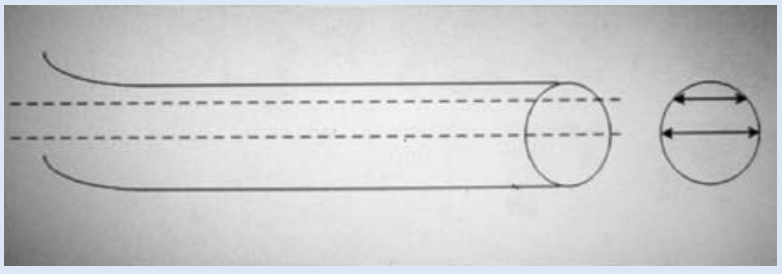

Fig. 3. Illustration of a horizontal, tubular structure. To get an accurate axial diameter of this structure, alignment should be through the centre of the longitudinal axis of the structure. Concentric or asymmetrical compression limits the ability to measure this on routine axial CT.

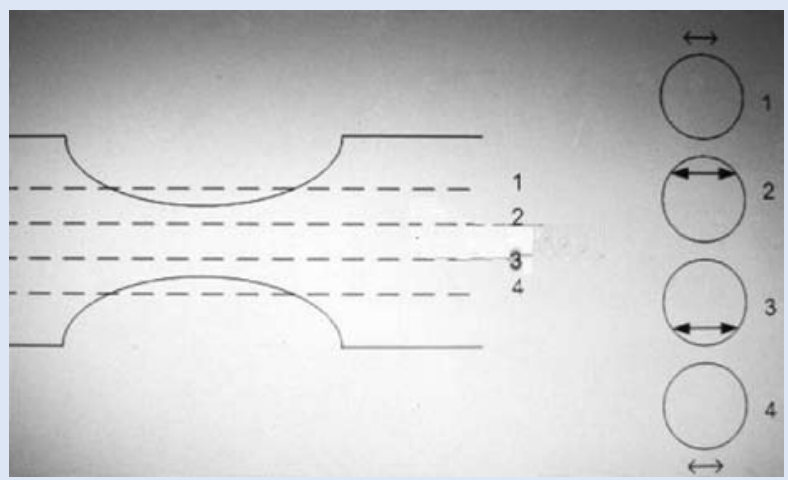

Fig. 4. Illustration of a partially compressed segment of a tubular structure. There is a higher possibility that cuts through the compressed segment might not be through the centre; therefore, an inaccurate diameter will be measured. There are at least 2 slices that may give a realistic diameter on the non-compressed segment and none in the compressed segment in this theoretical example.

- limited ability to detect subtle airway stenoses

- underestimation of the craniocaudal extent of the disease

- difficulty in displaying the complex 3-D relationships of the airway to adjacent mediastinal structures

- inadequate representation of airways oriented obliquely to the axial plane (Figs 2 - 4) 


\section{ORIGINAL ARTICLE}
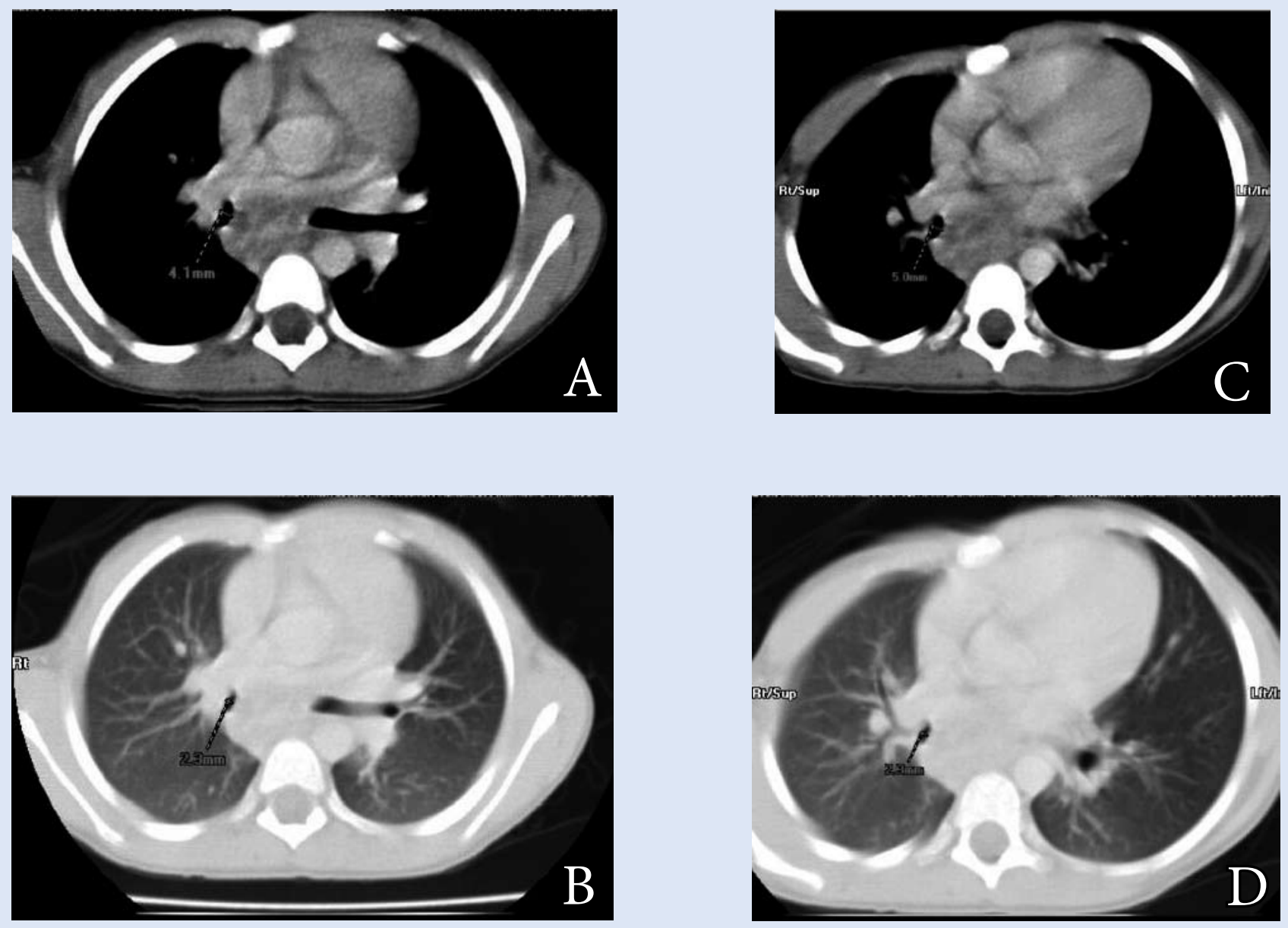

Fig. 5A - D. MDCT of the chest in a 52-month-old boy with compression of the bronchus intermedius owing to tuberculous lymphadenopathy. A: Standard axial image in soft tissue windows illustrating the measurement of the maximum stenosis in the bronchus intermedius (4.1 mm). Note the subcarinal lymphadenopathy. B: Measurement of the true diameter after MPR along the longitudinal axis (5.0 mm). C: Image A in lung windows measuring $2.3 \mathrm{~mm}$. $D$ : Image $B$ in lung windows measuring $2.3 \mathrm{~mm}$.

- difficulty in assessing the interfaces and surfaces of airways that lie parallel to the axial plane. ${ }^{8}$

Both axial CT and CT with MPR are accurate in detecting airway stenoses. ${ }^{5,9}$ Remy-Jardin et al. stated that MPR did not provide information that was not evident on transverse images. They did, however, find that mild airway narrowing was easier to recognize on MPR and the length of airway narrowing was easier to measure on MPR than on transverse CT images. ${ }^{9}$ Quint $e t$ al. found that CT with MPR occasionally provided information that was not evident on axial images, and advocated the use of MPRs in imaging of the central airways.

MPR to produce images of each of the major airways into its longitudinal axis, so as to obtain a true 'axial' diameter, is timeconsuming as it must be performed manually and individually for each bronchus as these are oriented uniquely in each patient. Measurements in the axial scan plane are more convenient and rapid as this is the routine image plane provided; i.e. there is no standard practice.

From the results of this study, no significant statistical difference was found between axial measurements and measurements taken after MPR providing the longitudinal axis, even in the relatively horizontal left main bronchus. The majority (56.4\%) of differences were $<0.4 \mathrm{~mm}$ with only $8.1 \%$ of the differences being $>1 \mathrm{~mm}$. Therefore, central airway stenoses are accurately detected and also not significantly overestimated or underestimated on axial images without MPR (Figs $5 \mathrm{~A}$ and 5B). It was, however, easier to evaluate mild stenoses and measure the length of stenoses with MPR.

Measurements taken in lung windows consistently measured the diameter of stenoses of the central airways less than soft tissue $(1.4 \mathrm{~mm}$ on average) (Figs 5C and 5D). The wide window and low-level setting of lung window should make it the desired setting for evaluating the airfilled airways. It is therefore more likely that soft tissue underestimates stenoses. In small-calibre airways, it is therefore important to measure stenoses on lung window to prevent underestimation.

Currently, a coarse parameter of $50 \%$ luminal obstruction of a main stem or lobar bronchus is considered a significant clinical indicator for management of children with airway obstruction. In the future, when there is less reliance on bronchoscopy and more reliance on imaging for airway narrowing, more refined measurement may become important to management decisions. 


\section{ORIGINAL ARTICLE}

\section{Conclusion}

The degree of central airway stenoses is accurately assessed on axial images, obviating the need to perform MPR to obtain a true axial diameter of the airways in children. Measurements taken on soft tissue window consistently underestimate stenoses.

1. Andronikou S, Joseph E, Lucas S, et al. CT scanning for the detection of tuberculous mediastinal and hilar lymphadenopathy in children. Pediatr Radiol 2004;34:232-236.

2. Kauczor HU, Wolcke B, Fischer B, Mildenberger P, Lorenz J, Thelen M. Three-dimensional helical CT of the tracheobronchial tree: Evaluation of imaging protocols and assessment of suspected stenoses with bronchoscopic correlation. Am J Roentgenol 1996;167:419-424.

3. Hartman TE, Primack SL, Lee KS, Swensen SJ, Muller NL. CT of bronchial and bronchiolar diseases. Radiographics 1994:14:991-1003.
4. Sorantin E, Geiger B, Lindbichler F, Eber E, Schimpl G. CT-based virtual tracheobronchoscopy in children - comparison with axial CT and multiplanar reconstruction: preliminary results. Pediatr Radio 2002;32:8-15

5. Quint LE, Whyte RI, Kazerooni EA, Martinez FJ, Cascade PN, Lynch JP 3rd. Stenosis of the central airways: Evaluation by using helical CT with multiplanar reconstructions. Radiology 1995;194:871-877.

6. Hoppe H, Dinkel HP, Walder B, von Allmen G, Gugger M, Vock P. Grading airway stenosis down to the segmental level using virtual bronchoscopy. Chest 2004;125(2):704-711.

7. Choe KO, Jeong HJ, Sohn HY. Tuberculous bronchial stenosis: CT findings in 28 cases. Am J Roentgenol 1990;155:971-976.

8. Boiselle PM, Ernst A. Recent advances in central airway imaging. Chest 2002;121:1651-1660.

9. Remy-Jardin M, Remy J, Deschildre E, Artaud D, Ramon P, Edme JL. Obstructive lesions of the central airways: evaluation by using spiral CT with multiplanar and three-dimensional reformations. Eur Radiol $1996 ; 6: 807-816$

\section{In Memoriam}

\section{Marie Grobbelaar}

31 July 1969 - 31 October 2009

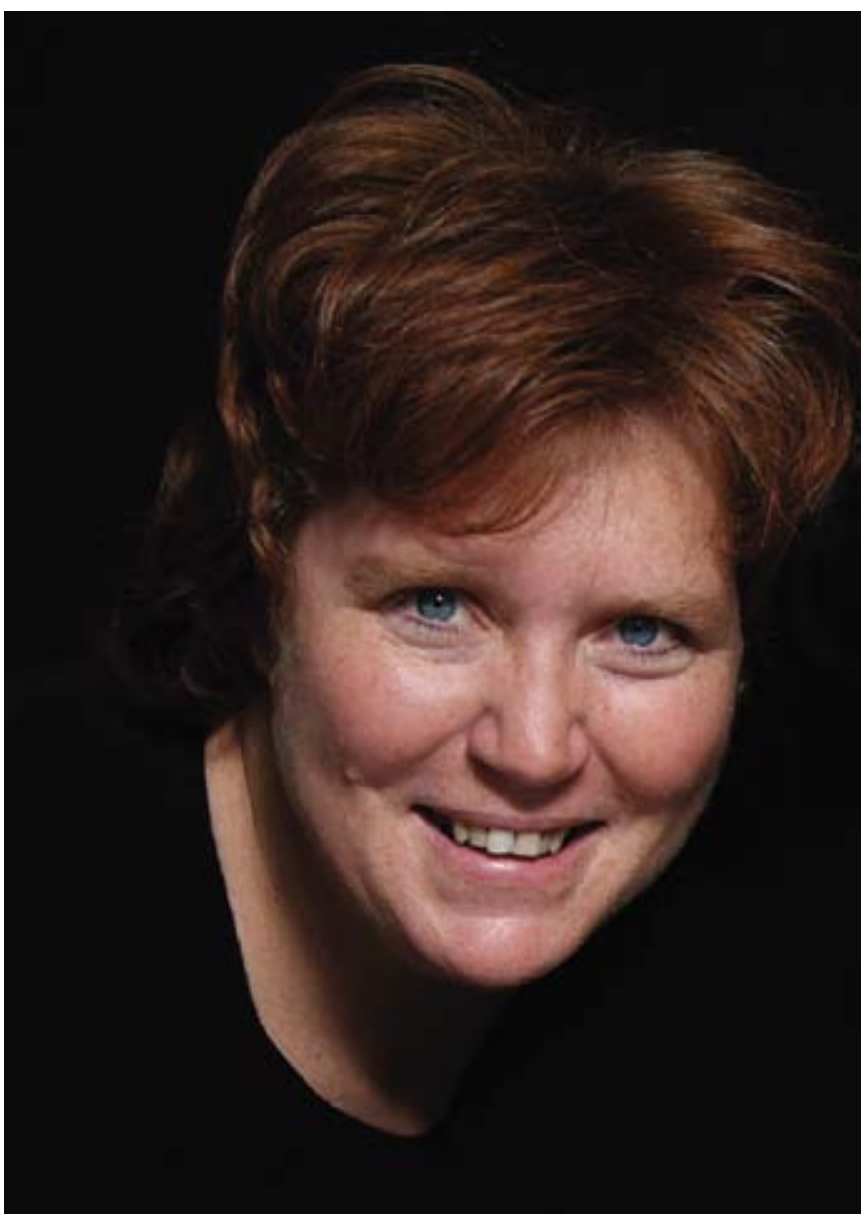

Almost a year since her passing, it is with great sadness that we think back on the life of our dear colleague and friend Marie. She completed her studies at Stellenbosch University in June 2008, and stayed on in the department as a senior specialist. She was a popular doctor, a favourite amongst all, and well known for her work ethic, enthusiasm, humility, caring nature and special sense of humour. She was a pillar to lean on, always making herself available and willing to go the extra mile. She had had intermittent headache for a week, was scanned on a Wednesday, a phaeochromocytoma was found, she came to work on the Friday, and passed away the next morning.

With the publication of her master's thesis in this issue of the $S A J R$, we salute and honour this unique and beautiful soul. We extend our deepest sympathy to her husband Johan and their children Chris and Jenna.

\section{Consultants and registrars}

Department of Diagnostic Imaging

Stellenbosch University 